\title{
A tabu search approach to the cargo prioritisation and terminal allocation problem
}

\section{Jingjing Tong*}

Department of Engineering and Technology, Southeast Missouri State University

One University Plaza - MS6825, Cape Girardeau, MO 63701, USA

Email: jtong@semo.edu

*Corresponding author

\section{Heather Nachtmann}

Maritime Transportation Research and Education Center (MarTREC),

College of Engineering,

University of Arkansas,

4184 Bell Engineering Center,

Fayetteville, AR 72701, USA

Email: hln@uark.edu

\begin{abstract}
To mitigate inland waterway disruption impacts, we develop a tabu search (TS) approach to solve the cargo prioritisation and terminal allocation problem (CPTAP) to minimise the total value loss of disrupted barge cargoes by offloading the cargoes to the land transportation modes. CPTAP is formulated as a nonlinear binary integer program, and problems of realistic size can be efficiently and effectively solved with an efficient heuristic approach. Given different neighbourhood structures, multiple TS variants are attempted and compared. Solving CPTAP with our TS heuristic leads to the lowest cargo value loss and the shortest response time for the disrupted barges compared to a genetic algorithm approach and a naïve minimise distance strategy. The CPTAP model and the TS heuristic provide the inland waterway stakeholders timely information and suggestion on developing a practical and effective reaction plan when the inland waterway is closed due to natural disasters or manmade events.
\end{abstract}

Keywords: metaheuristics; OR in maritime industry; inland waterway transportation; cargo prioritisation.

Reference to this paper should be made as follows: Tong, J. and Nachtmann, H. (2020) 'A tabu search approach to the cargo prioritisation and terminal allocation problem', Int. J. Shipping and Transport Logistics, Vol. 12, No. 3, pp.147-173.

Biographical notes: Jingjing Tong is an Assistant Professor of Industrial Engineering within the Department of Engineering and Technology at Southeast Missouri State University. She received her $\mathrm{PhD}$ in 2014 from the Department of Industrial Engineering at the University of Arkansas. Her teaching and research focus on applied operations research in transportation, logistics, supply chain, disruption response and homeland security, and engineering decision analysis. 
Heather Nachtmann is the Associate Dean for Research in the College of Engineering, Earl J. and Lillian P. Dyess Endowed Chair in Engineering and a Professor of Industrial Engineering at the University of Arkansas. She serves as the Director of the Maritime Transportation Research and Education Center (MarTREC), a US Department of Transportation University Transportation Center and the Mack-Blackwell Transportation Center. Her research includes economic and operations analysis of inland waterways, intermodal network optimisation, and vulnerability assessment and emergency preparedness for transportation networks. She is a Fellow of the American Society for Engineering Management and Institute of Industrial and Systems Engineers, and Co-Editor-in-Chief of the Engineering Management Journal and an associate editor for The Engineering Economist. She received her $\mathrm{PhD}$ from the University of Pittsburgh.

\section{Introduction}

Composed of waterways, rivers, locks and dams, canals, and bridges, the 12,000 navigable miles of US' inland waterway system (USACE, 2012a) is a crucial transportation mode for moving large quantities of bulk cargo to their destinations. The vast inland waterway transportation system serves thirty-eight States with four major navigation channels - Mississippi River, Ohio River, Gulf Intercoastal Waterway, and Pacific Coast System (ASCE, 2009). In 2013, a total of 2.3 billion tons of domestic and international freight was transported by water (USACE, 2013). Measured by percent of total inland waterborne tonnage, the major commodities transported on the inland waterways are petroleum (41\%), coal (14\%) and food and farm products (12\%) (USACE, 2013). In addition to its low transportation rate, barge transportation is recognised as an environment-friendly and capacity-efficient transportation mode that reduces surface transportation congestion and improves the air quality.

As described in paper from the Tong and Nachtmann (2017), an unexpected disruption to the inland waterway transportation system due to a natural disaster, vessel accident, or terrorist attack may result in a non-navigable water level or destruction of major navigation infrastructure (e.g., bridges, locks and dams) that shuts down the navigation channel and requires barge cargoes to be offloaded and transported to their final destination via an alternative land-based transportation mode. A barge tow typically consists of nine to 15 barges, each with the capacity to carry approximately sixty truckloads or 15 railcar loads of cargo. The disrupted cargo may exceed the existing capacity of accessible terminals and alternative modes of transportation, and the cargo's value diminishes in terms of economic value, societal benefit, and customer satisfaction as response time elapses.

This paper presents a tabu search (TS) approach to the cargo prioritisation and terminal allocation problem (CPTAP) which was introduced by Tong and Nachtmann (2017) and minimises the total value loss by optimally prioritising disrupted barges through consideration of multiple prioritisation factors including commodity type, cargo value, terminal capacity, and barge draft. CPTAP is a combinatorial optimisation problem that cannot be solved by an exact solution approach under realistic problem size conditions. In our previous work, we formulated CPTAP as a nonlinear binary integer program, and problems of realistic size were efficiently and effectively solved with a 
genetic algorithm approach (Tong and Nachtmann, 2017). The details of CPTAP and its similarities to the berth allocation problem (BAP) (see original work by Imai et al., 1997, 2001, 2003) are discussed in the paper by Tong and Nachtmann (2017). CPTAP is a three dimensional assignment problems that involves two decisions, barge/ship-to-terminal/berth assignment and offload/service order at the terminal/berth, and the elapsed time of a barge/ship that is incorporated into the objective function partially depends on its predecessors (Tong and Nachtmann, 2017).

We were motivated to explore TS as a solution approach to CPTAP primarily for two reasons:

1 Two principles guide the development of metaheuristics, population search and local search (Cordeau et al., 2002). Our prior work employed a population search strategy, a GA-based heuristic that recombines a number of parent solutions to generate child solutions. The TS heuristic is a local search strategy which obtains new solutions through a neighbourhood search. Our investigation of the CPTAP TS heuristic will reveal the performance of a local search solution approach to CPTAP and enable us to compare these two principles for CPTAP in terms of the solution quality, computational efforts and robustness. Our interest in comparing the two principles also contribute to our decision of not choosing simulated annealing to solve CPTAP, although it was very effective in solving the similar berth scheduling problem by Kim and Moon (2003).

2 CPTAP has similarities to BAP, and TS has been successfully applied to BAP as evidenced by the literature (Cordeau and Laporte, 2004; Meisel and Bierwirth, 2009; Giallombardo et al., 2010).

As a local search metaheuristic, TS examines the solution space by conducting a neighbourhood search based on the current solution, picking up the best found solution according to a penalised cost function, and then searching the neighbourhood of the new solution. The new solution may not be a feasible solution but could be admitted to allow for exploration of its neighbourhood space. Cycling of a set of solutions may occur since the selection of current solution does not follow a fixed path such as increasing/decreasing objective function values. Therefore, a tabu mechanism is used to store the solution modifications in previous steps, and these modifications are not allowed in the next couple of iterations in order to avoid exploring investigated space repeatedly (Bräysy and Gendreau 2005; Taillard et al., 2001). TS heuristic has been widely applied to many problem settings, among which the vehicle routing problem (VRP) is one of the most popular problems where TS is implemented as a solution approach.

The contribution of this work is to develop and evaluate a TS heuristic that comprises its characteristics discussed above for a relatively new problem - CPTAP. We identify a most suitable TS approach for CPTAP, the Unified TS heuristic (Cordeau et al., 2001), among the many TS heuristics found in literature. We present three neighbourhood structures for the TS and examine which is most efficient for solving CPTAP in terms of solution quality and computation time. In addition we compare our best TS CPTAP approach to two other cargo prioritisation strategies [CPTAP solved by GA heuristic (Tong and Nachtmann, 2017) and a simple minimise distance strategy] and verify the effectiveness of the TS CPTAP solution approach. 
The structure of the paper is organised as follows: Section 2 provides the detailed description of the CPTAP and introduces the mathematical model of the problem. Sections 3 and 4 summarise the relevant TS literature, present a flow chart of the proposed heuristic and describe its major components. Section 5 and 6 respectively discuss the parameter setting and experimental work for our TS heuristic. Section 7 compares the multiple cargo prioritisation strategies. We conclude the work in Section 8 and discuss future work in this area.

\section{CPTAP Description and Model Formulation}

As previously described in Tong and Nachtmann (2017), CPTAP is graphically represented in Figure 1 through the depiction of a recent inland waterway disruption event:

- On 20 January 2014, a railroad bridge over the Arkansas River suffered a mechanical failure which halted all barge traffic on that section of the river (Magsam and McGeeney, 2014). Five locks and dams (L/Ds) serve that river section, and ten terminals are located along both sides of the river (locations shown in Figure 1). Each terminal is capable of offloading specific commodity types of cargo depending on its handling facilities.

- According to the USACE Lock Performance Monitoring System (2014) eight barge tows, commonly consisting of nine to 15 barges each, were travelling up and down the disrupted river section at the time of the event as shown in Figure 1. Since the disruption prohibits barge traffic at the bridge location, the six barge tows (consisting of approximately 60 disrupted barges) that are travelling towards and beyond the damaged bridge (shaded in black) are disrupted and need to be prioritised and redirected through implementation of CPTAP. The two barge tows that have already passed under the damaged bridge and are travelling away from the disruption point (shaded in white) are not impacted.

- Since the disrupted barges are no longer able to travel to their original designation along the disrupted inland waterway, CPTAP determines an accessible terminal for offloading and rerouting the cargo on each disrupted barge and the barge's offload order at the designated terminal since more than one barge may be sent to a given terminal. Because the disruption has effectively divided the inland waterway into two sections, CPTAP is typically employed twice, once for disrupted barges located on the river above the disruption and once for disrupted barges located on the river below the bridge.

The widely-studied BAP shares a similar decision structure to our CPTAP, and the original work in BAP supported the development of our model formulation (Imai et al., 1997, 2001, 2003; Nishimura et al., 2001). The focus of CPTAP is to assign barges to terminals with consideration of cargo offloading priorities at a terminal while BAP assign vessels to berths with consideration of vessel ordering at a berth. We adopt the three dimensional decision variable that is a common variable type in BAP papers and adapt some of the constraints found in BAP literature (Imai et al., 1997, 2001, 2003; Nishimura et al., 2001) to develop our CPTAP model formulation [constraint sets (2), (3), (4) and (7)]. 
Figure 1 Arkansas River disruption

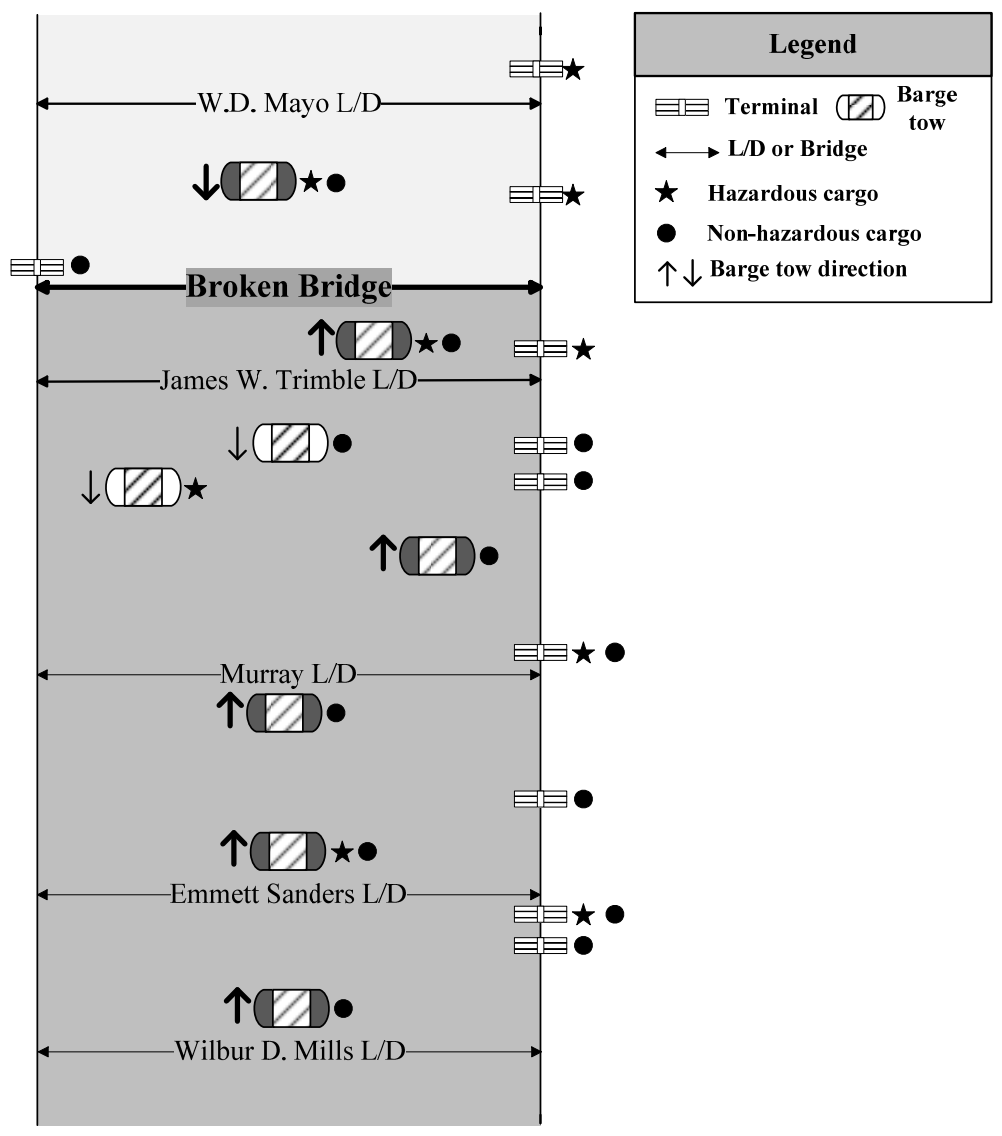

Source: Tong and Nachtmann (2017)

The sets, variables and parameters of CPTAP formulation are described as follows (Tong and Nachtmann, 2017):

Sets

$J \quad$ Set of barges with non-hazardous cargo.

$H$ Set of barges with hazardous cargo.

I Set of real terminals.

$D$ Set of dummy terminal (one).

$K$ Set of barge orders at a given terminal.

$N$ Set of commodity cargo types.

Decision variables

$x_{i j k} \in\{0,1\} \quad 1$ if barge $\mathrm{j}$ is assigned to terminal $i$ in the $k$ th order; 0 otherwise. 


\section{Parameters}

$t_{i j} \quad$ water transport time of barge $j$ from its location at the time of disruption to terminal $i$

$h_{i j} \quad$ handling time of barge $j$ at terminal $i$

$a_{i j} \quad$ actual contributing time of barge $j$ that is assigned to terminal $i$ in the $k$ th order

$r_{i j} \quad$ land transport time of barge $j$ cargo from terminal $i$ to its final destination by alternative mode of transportation

$\alpha_{j} \quad$ value decreasing rate of barge $j$ cargo per unit volume per unit time

$u_{i n}$ offload capacity for cargo $n$ at terminal $i$ during the disruption response

$c_{j} \quad$ cargo volume on barge $j$

$w_{i} \quad$ water depth at terminal $i$

$d_{j} \quad$ draft depth of barge $j$

$e_{j n} \quad 1$ if barge $j$ carries cargo $n$; 0 otherwise

$s \quad$ safety level

$v_{j} \quad$ total value of barge $j$ cargo

$p \quad$ sinking threshold.

$$
a l i j= \begin{cases}t_{i j}+h_{i j} & k=1 \\ h_{i j} & k \neq 1 t_{i j} \leq \sum_{m \in J \cup H} \sum_{k \in K-1} a_{i m} \\ t_{i j}+h_{i j}-\sum_{m \in J+H} \sum_{k \in K-1} a_{i m} & k \neq 1 t_{i j}>\sum_{m \in J \cup H} \sum_{k \in K-1} a_{i m}\end{cases}
$$

The actual contributing time is defined as the amount of water transport and handling time incurred by assigning and offloading a barge at the terminal. As shown in equation (1), when a barge is assigned the first offload turn, its actual contributing time $a_{i j}$ is the sum of its water transport time $t_{i j}$ and its handling time $h_{i j}$. For the barges assigned to the second or later offload turn at a given terminal:

Case 1 When a barge arrives to its assigned terminal before barges with earlier offload turns complete their water transportation and offloading, it must wait until any barge(s) with higher priority arrives the terminal and completes the offloading before the barge can start its own. Therefore, its actual contributing time reduces to its handling time $h_{i j}$.

Case 2 When a barge arrives to its assigned terminal after any barge(s) with higher priority completes its water transportation and offloading, its actual contributing time is the sum of its water transport time $t_{i j}$ and handling time $h_{i j}$ minus the cumulative actual contributing time of the preceding barge(s) (Tong and Nachtmann, 2017).

The CPTAP is formulated as a nonlinear integer program (NLIP) as follows (Tong and Nachtmann, 2017): 
Min

$$
\begin{aligned}
& \sum_{i \in I} \sum_{j \in J \cup H} \sum_{k \in K}\left\{\left(\sum_{m \in J \cup H} \sum_{k \in K} a_{i m} x_{i m(k-1)}+a_{i j}+r_{i j}\right) c_{j} \alpha_{j} x_{i j k}\right\} \\
& +\sum_{i \in D} \sum_{j \in J} \sum_{k \in K} v_{j} x_{i j k}
\end{aligned}
$$

s.t.

$\sum_{i \in I \cup D} \sum_{k \in K} x_{i j k}=1 \quad \forall j \in J$

$\sum_{i \in I} \sum_{k \in K} x_{i j k}=1 \quad \forall j \in H$

$\sum_{i \in J \cup H} x_{i j k} \leq 1 \quad \forall i \in I, k \in K$

$\sum_{j \in J \cup H} x_{i j k} \geq \sum_{j \in J \cup H} x_{i j(k+1)} \quad \forall i \in I \cup D, k \in K /|K|$

$\sum_{j \in J \cup H} \sum_{k \in K} c_{j} e_{j n} x_{i j k} \leq u_{i n} \quad \forall i \in I \cup D, n \in N$

$\sum_{i \in I \cup H} \sum_{k \in K}\left(w_{i}-d_{j}\right) x_{i j k} \geq s \quad \forall j \in J \cup H$

$$
\sum_{i \in I} \sum_{k \in K}\left\{\left(\sum_{m \in J \cup H} \sum_{k \in K} a_{i m} x_{i m(k-1)}+a_{i j}+r_{i j}\right) c_{j} \alpha_{j} x_{i j k}\right\} \leq v_{j} p \quad \forall j \in J \cup H
$$

$$
x_{i j k} \in\{0,1\} \quad \forall i \in I \cup D, k \in K, j \in J \cup H
$$

Constraint set (2) ensures that the barge with non-hazardous cargo either is offloaded in some priority order at a terminal or stays on the inland waterway. Constraint set (3) guarantees that all barges with hazardous cargo must have a designated terminal to offload their cargo. Constraint set (4) ensures that no more than one barge is offloaded at a terminal at a priority turn. Constraint set (5) aesthetically ensures that the priority order at each terminal starts from the first priority turn. Constraint set (6) indicates that the total amount of a particular cargo offloaded at the terminal does not exceed the terminal capacity. Constraint set (7) ensures that the barge draft plus the safety level cannot exceed the water depth at the terminal. Constraint set (8) ensures that the total value loss of the barge cargo that is transported for offloading to an alternative transportation mode is less than or equal to the product of the sinking threshold and the total cargo value. We will not reroute the cargo to an alternative mode if doing so leads to significant value loss (Tong and Nachtmann, 2017). Constraints that are actively involved into the TS process are the capacity constraint set (6), draft constraint set (7), and value loss constraint set (8).

\section{Literature review}

We investigated papers that employ a TS heuristic to solve the BAP and VRP. The BAP TS literature was most valuable in developing our TS heuristic since it has the similar framework with CTPAP. However, since a limited number of BAP papers focus on the TS heuristic, we extended our literature review to include the VRP literature because considerable papers have investigated TS implementation in VRP. 


\subsection{TS in BAP}

Cordeau and Laporte (2004) proposed a new formulation approach for the discrete berth allocation problem (BAP) - the multi-depot VRP with time windows (MDVRPTW) formulation which handles the weighted sum of the service times and the time windows of the berthing times. They employed a TS heuristic to solve the discrete case with an extension for the continuous BAP, which is capable of obtaining optimal solutions for small size instances and better solutions for large size instances when compared to a truncated branch-and-bound algorithm. Meisel and Bierwirth (2009) integrated the BAP and crane assignment problem (BACAP) to provide an integer linear program model that incorporates the practical impact of the crane resources on the handling time. Both squeaky wheel optimisation and TS heuristic are employed and compared in solving a set of benchmark problems. Giallombardo et al. (2010) studied the BACAP as a mixed integer linear program formulation where TS is used to solve their BAP decision (adapted from Cordeau and Laporte, 2004) and obtains good solutions within a satisfactory amount of time.

\subsection{TS in VRP}

A steady, thorough, and extensive evolution of VRP heuristics has been observed in the last forty years, among which the TS heuristic is identified as one of the best metaheuristics for the VRP (Cordeau and Laporte, 2004; Taillard et al., 2001). More than fifty papers have been published on this topic since the first TS implementation to the VRP in 1989 (Laporte, 2009). Multiple survey papers have summarised the TS literature in VRP (Eksioglu et al., 2009; Laporte, 2009; Braysy and Gendreau, 2005; Cordeau et al., 2002; Cordeau and Laporte, 2004) and identified TS as a competitive metaheuristic method to solve VRP. Some researchers consider TS to be the best metaheuristic method for solving the VRP (Cordeau et al., 2002). Nine papers were found to be the most informative to our work and are summarised in Table 1. Among these TS heuristics, the Unified TS is chosen as the most suitable TS method for CPTAP due to its proved efficiency, robustness (small number of parameters to be determined), and compatibility to our CPTAP structure.

\section{Tabu search heuristic for CPTAP}

In this section, we describe our CPTAP TS heuristic. The general CPTAP TS framework is developed from the Unified TS proposed by Cordeau et al. (2001). We adapt their heuristic according to the characteristics of CPTAP and consider additional heuristic design components including two potential initial solution generation approaches based on the CPTAP solution structure, three possible neighbourhood structures to select the best neighbourhood scheme, two alternative tabu management approaches, and possible incorporation of a post-optimisation step utilising a local swap structure. 
Table 1 Comparison of select TS VRP literature






\subsection{CPTAP tabu search heuristic flowchart}

Figure 2 presents a flowchart of the CPTAP TS heuristic that is developed from adapting the unified TS (Cordeau et al., 2001).

Figure 2 CPTAP TS flowchart

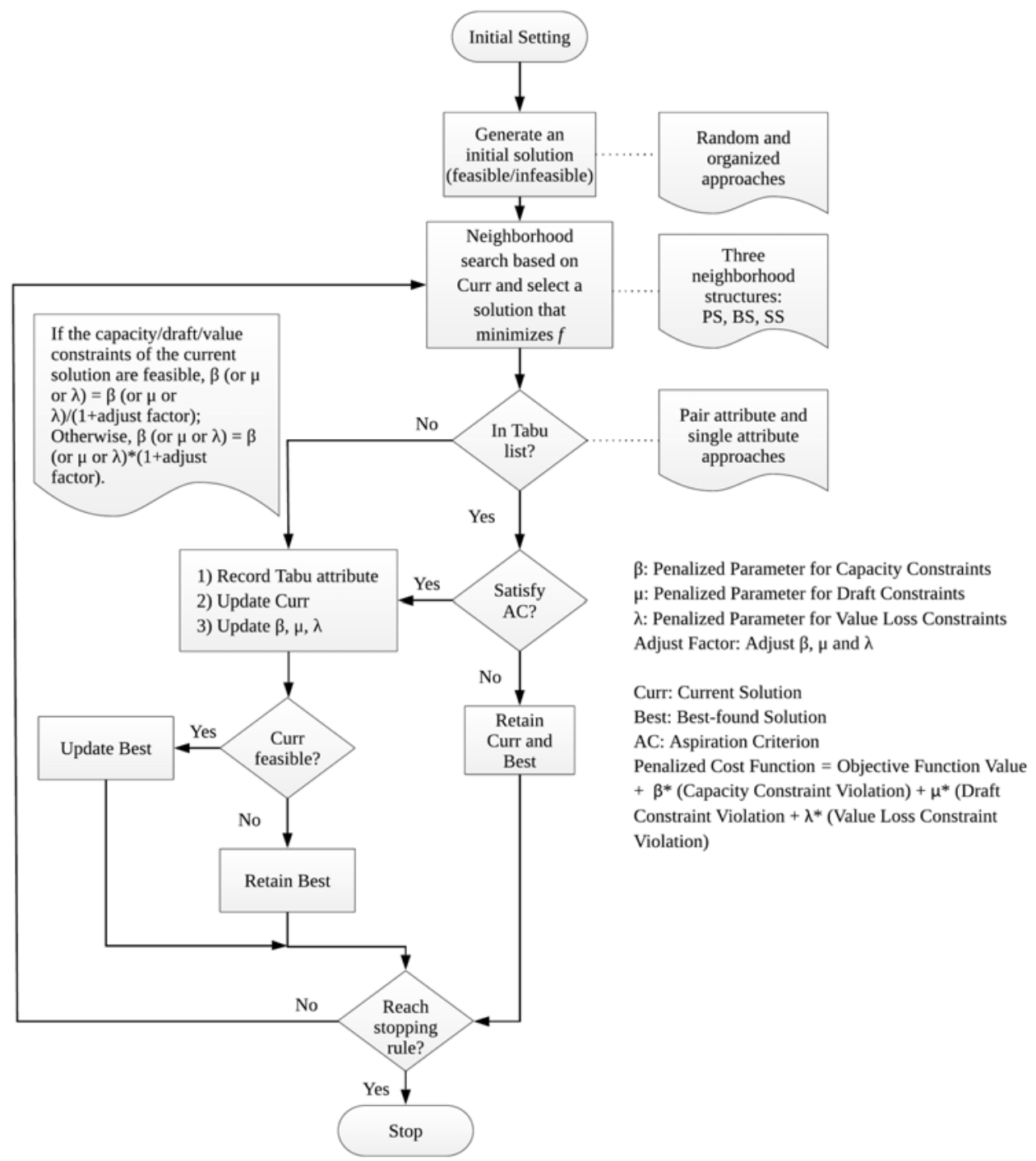

\subsection{Initial solution}

An initial solution is required to start the CPTAP TS search process. This initial solution may be found to be infeasible since our heuristic explores feasible and infeasible solution spaces. Two methods for generating an initial solution are developed and compared: 
random generation (randomly produces a solution without any restriction on the solution structure) and organised generation [attempts to find a 'near-feasible' initial solution that meets the draft constraints (constraint set (7)] and is not likely to violate the capacity constraints constraint set (6). The organised generation approach is more likely to quickly generate a feasible initial solution and is described below:

1 Record the acceptable barges for each terminal in terms of barge draft restriction:

- For each real terminal $i$, assign the barges that the terminal can accept according to the draft constraint.

- For dummy terminal $d$, assign the barges that carry non-hazardous cargo to the dummy terminal.

2 For $i=1, \ldots, I-1$, conduct the following processes:

- Determine the type of cargo each barge carries.

- If adding the next barge will cause the capacity violation of a specific cargo type, remove the barge from the assigned terminal; otherwise, keep the barge.

3 Delete the duplicate barges that have appeared in the previous terminals.

Preliminary experimentation indicated that the random generation produces better CPTAP solutions compared to organised generation when controlling for the other TS constituents and parameters. Therefore, we will select random generation as the initial solution generation approach for our CPTAP TS heuristic.

\subsection{Penalised cost function}

In the unified TS (Cordeau et al., 2001) a penalised cost function is used to evaluate solutions as a replacement for the objective function. Solution $x$ represents a decision result from CPTAP that could be feasible or infeasible. $f(x)$ denotes the original objective function value (i.e., the total value loss of the disrupted barge cargo); $c(x), d(x)$ and $v(x)$ denote the total violation of the constraint sets - capacity constraint [constraint set (6)], draft constraint [constraint set (7)] and value loss constraint [constraint set (8)]. The violated amount of each constraint set is added to the objective function to form the penalised cost function as follows:

$$
P(x)=f(x)+\beta c(x)+\mu d(x)+\lambda v(x)
$$

where

$$
\begin{aligned}
& f(x)=\sum_{i \in I} \sum_{j \in J \cup H} \sum_{k \in K}\left\{\left(\sum_{m \in J \cup H} \sum_{k \in K} a_{i m} x_{i m(k-1)}+a_{i j}+r_{i j}\right) c_{j} \alpha_{j} x_{i j k}\right\} \\
& +\sum_{i \in D} \sum_{j \in J} \sum_{k \in K} x_{i j k} t v_{j} \\
& c(x)=\sum_{i \in I \cup D} \sum_{n \in N} \max \left(\sum_{j \in J \cup H} \sum_{k \in K} c_{j} m_{j n} x_{i j k}-u_{i n}, 0\right) \\
& d(x)=\sum_{j \in J \cup H} \max \left\{s-\sum_{i \in I} \sum_{k \in K}\left(w d_{i}-d_{j}\right) x_{i j k}, 0\right\}
\end{aligned}
$$




$$
\begin{aligned}
v(x) & =\sum_{j \in J \cup H} \max \left\{\sum_{i \in I} \sum_{k \in K}\left\{\left(\sum_{m \in J \cup H} \sum_{k \in K} a_{i m} x_{i m(k-1)}+a_{i j}+r_{i j}\right) c_{j} \alpha_{j} x_{i j k}\right\}\right. \\
& \left.-t v_{j} p, 0\right\}
\end{aligned}
$$

$\beta, \mu$ and $\lambda$ are positive self-adjusting parameters.

Functions $c(x), d(x)$ and $v(x)$ assure that only the violated amounts of the infeasible constraints are penalised in the cost function. The function values of $c(x), d(x)$ and $v(x)$ for a feasible solution are equal to zero. In order to diversify the search space, parameters $\beta, \mu$ and $\lambda$ are updated by a positive factor $\delta$ according to the current solution. If the current solution is feasible with respect to capacity/draft/value loss constraints, $\beta(\mu$ or $\lambda)=\beta(\mu$ or $\lambda) /(1+\delta)$; otherwise, $\beta(\mu$ or $\lambda)=\beta(\mu$ or $\lambda) \times(1+\delta)$. Explanation of this diversification step is that we tend to penalise the constraint set lightly once a feasible constraint of that constraint set appears. This is because we have reached a feasible area associated with the constraint set so we should extend the search space by allowing the search to reach more infeasible space. On the other hand, if the constraint is infeasible at a given iteration, it means we are already searching the infeasible space relating to that constraint. To ensure we return to a feasible space to produce an acceptable solution, we increase the penalty cost to force the search back to a feasible solution. This updating process is carried out at the end of each iteration in order to adapt the search at the next iteration.

\subsection{Neighbourhood structure}

Neighbourhood search is an important step in any TS heuristic as it determines the transition between the current solutions of different iterations (Ceschia et al., 2011). Several papers employing the unified TS incorporate an insertion step to complete their neighbourhood search (Cordeau et al., 1997, 2001). Based on the features of our CPTAP model, we consider the following four neighbourhood search methods:

a Partial shift (PS): this neighbourhood move is defined by removing a barge $j$ from the current assigned terminal $i$ and reinserting it to another terminal $i^{*}$ with a random prioritisation order $k$ given at the terminal $i^{*}$. New current solution candidate set includes moving each barge $j$ to each terminal $i^{*}$ (other than the original assigned terminal $i$ ). The prioritised order at the new terminal is randomly generated in order to reduce the computational effort. Barge $j$ can be reinserted with any order at the last terminal (the dummy terminal) because there is no actual prioritisation for barges that stay on the disrupted waterway.

b Blind SWAP search (BS): this move takes a liberal perspective on neighbourhood search by randomly exchanging two components in the current solution irrespective of their representation of barge or terminal. BS conducts the exchange on a predetermined number of SWAP pairs.

c Switch SWAP search (SS): adapted from BS Search, SS Search exchanges two barges at two different terminals, and the two barges take each other's priority turn as the insertion location. SS conducts the exchange on a predetermined number of SWAP pairs. 


\subsection{Tabu management and aspiration criterion}

In TS, solutions with certain attributes are prohibited from a certain number of iterations in order to avoid cycling around a local minimum (Braysy and Gendreau 2005). The selection of the attribute (also called tabu management) is critical since it influences the solution selection. Two tabu management approaches are considered in the development of our CPTAP TS heuristic: pair attribute and single attribute:

a Pair attribute: two elements are included in the Pair Attribute approach. The attribute structures are different for the Shift (PS) and SWAP (BS and SS) neighbourhood searches as follows:

- For PS: $A(x)=\{(i, j)$ : barge $j$ is offloaded at terminal $i\}$. When a neighbourhood move of removing barge $j$ from terminal $i$ and inserting $j$ into terminal $i^{*}$ is completed, the attribute $(i, j)$ is declared $t a b u$, which means barge $j$ cannot be reassigned to be offloaded at terminal $i$ for a predefined number of iterations.

- $\quad$ For BS and SS: $A(x)=\left\{\left(j, j^{\prime}\right)\right.$ : barge $j$ and $j^{\prime}$ are exchanged $\}$. Similarly, if attribute $\left(j, j^{\prime}\right)$ is recorded in the tabu list, it means the two barges cannot be switched for the next certain number of iterations. A special case in BS is to exchange a value zero (for terminals) instead of a nature number (for barges). Since there are multiple zeroes representing different terminals, we do not include the pair attribute into the tabu list if one or both exchanging components are zero.

b Single attribute: different from the pair attribute that considers two elements in a pairwise fashion, single attribute maintains the records of the two elements separately. For example, the pair attribute for PS neighbourhood search is $A(x)=\{(i, j)$ : barge $j$ is offloaded at terminal $i\}$. The translation in single attribute is $A(x)=\{(i)$ and $(j)$ : barge $j$ is offloaded at terminal $i\}$, which means that any solution that relates to the move of barge $j$ or the insertion at terminal $i$ in the following number of iterations based on the tabu list length will not be selected as the current solution.

Preliminary experimentation suggests that the pair attribute approach performs better than the single attribute in influencing CPTAP solution quality. Therefore, we select the pair attribute tabu management approach for the CPTAP TS heuristic.

No matter which attribute is adopted, tabu can be overruled by the aspiration criterion that allows a tabued solution to be accepted. Various definitions of aspiration criterion are introduced in literature, e.g., improving the current best solution (Nguyen et al., 2013) or improving the best feasible/infeasible solution yet found (Brandão, 2009). In our CPTAP TS heuristic, we define the aspiration criterion as a prohibited move is revoked if it is better than the current optimal solution (the current best feasible solution found). Our employed aspiration criterion ensures that we do not miss a 'good' feasible solution that may be hidden by the tabu scheme.

\subsection{Post-optimisation step}

After obtaining the best-found solution $s^{*}$ through TS procedures, we consider a post-optimisation Local SWAP step after the selection of parameter values and the best 
neighbourhood structure to potentially find a better feasible solution. For a best-found solution $s^{*}$ (which is feasible), several pairs of barges at each terminal are interchanged to produce a new solution $s^{\prime}$. Since the assignment of barges to a terminal is not changed, the capacity and draft constraints are guaranteed to be feasible. However, the new assignment may violate value loss constraints. Therefore, the value loss constraints are checked for the new solution $s^{\prime}$. If the solution is still feasible and the new solution $s$ ' produces better penalised cost function value, the original best-found solution $s^{*}$ will be replaced by the new solution $s$ '. Preliminary experimentation suggests a very slight decrease of the objective function value which does not support further consideration of incorporating a post-optimisation step into the CPTAP TS heuristic.

\section{Parameter analysis}

In this section we set the parameter values of our CPTAP TS heuristic systematically through a one-way sensitive analysis in order to maximise the heuristic's performance. Since the penalty parameters $\beta, \mu$ and $\lambda$ will be modified frequently by adjustment factor $\delta$, we focus the analysis on the adjustment factor instead of the initial parameter values. Based on preliminary analysis, the initial penalty parameters $\beta, \mu$ and $\lambda$ are set to 10,100 , and 10. A sequential parameter determination approach is employed in the following sequence:

a adjustment factor $\delta$ of the penalty parameters

b length of tabu list

c number of iterations.

Once a parameter's value is set, it is adopted for the reminder of the analysis. As we discussed in Section 4, random generation and pair attribute are employed as the initial solution generation approach and the tabu management method respectively. The size of CPTAP problem instances are classified into small (five terminals and five/seven/nine barges), medium (ten terminals and thirty barges), and large (15 terminals and fifty barges). Preliminary analysis indicates that the CPTAP TS heuristic performance on small-sized problems is not sensitive to changes in the parameter settings so we limit our discussion to our sensitivity analysis on medium and large size problems.

\subsection{Adjustment factor}

Based on our preliminary experiments and related literature (Cordeau et al., 1997; Gendreau et al., 2008), the appropriate value of $\delta$ should vary within the range $[0.01,5]$. If the value of $\delta$ is too small, the heuristic cannot deliver a feasible solution if the search locates a good infeasible area. On the other hand, if the value of $\delta$ is too large, the search jumps drastically around the solution area and may prevent the search from investigating consecutive solutions. We consider six values $(0.01,0.05,0.1,0.5,1$ and 5$)$ within the interval $[0.01,5]$. Ten medium size instances are run ten times under each of the six values of $\delta$, resulting in 100 solutions for each $\delta$ value. Maximum, minimum, and average objective function (total value loss) results for each $\delta$ value are summarised in the stock charts shown in Figure 3. The upmost point and the down most point of each vertical line indicate the maximum and minimum solutions among the 100 solutions, while the marker 
in the middle represents the average solution result. According to Figure 3 results for the medium size instances, the average values obtained when $\delta$ is set at 1 or 5 is undesirably higher than those found by the lower $\delta$ values. Therefore, we reduce the parameter candidate pool to those that fall between 0.01 and 0.5 . Appendix 1 displays detailed maximum, minimum, and average objective function results of the medium size problem. When $\delta$ is set to 0.05 , there are more instances that have the lowest minimum and average results (italic values in Appendix 1). In addition, Figure 3 for the medium size instances shows that $\delta$ equal to 0.05 produces the smallest solution variance. Therefore, we select 0.05 as the adjustment factor value for medium size problems. The same experiments were conducted on large size CPTAP instances, and we draw the same conclusion that $\delta$ should be set to 0.05 (see large size results in Figure 3 and Appendix 1).

Figure 3 Sensitive analysis results of adjustment factor $\delta$, (a) middle size (avg) (b) large size (avg)

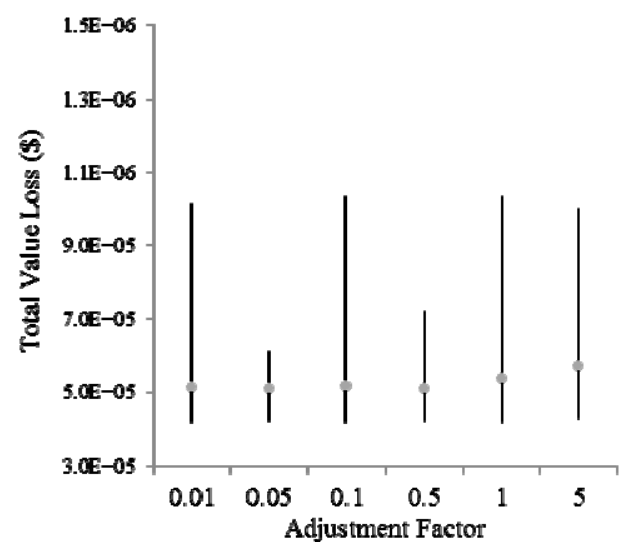

(a)



(b)

\subsection{Length of tabu list}

We also conduct a one-way sensitivity analysis on ten instances of medium and large size problems to investigate the length of the tabu list that produces the best quality solutions. Based on preliminary experimentation, we set the tabu list interval as [20, 80] and considered four values as the candidate tabu list lengths $(20,40,60$, and 80). Each instance is run 10 times for each tabu list length value. The average and minimum objective function results among ten runs are calculated for each instance with each candidate value. Figure 4 summarises the CPTAP TS heuristic performance for each tabu list length. Because CPTAP is a minimisation problem, we want the parameter value that delivers the most instances with minimum objective function value and the minimum average objective function value at the same time. We observe that 20, 40, and 60 tabu list lengths are on the Pareto frontier for the medium size instances, and 20 is the only Pareto point for the large size instances. Combining the results from the two scenarios displayed in Figure 4, a tabu list length of 20 is selected in order to find the best solution (minimum objective function value) and stay robust (minimum average objective function value). 
Figure 4 Sensitive analysis of the length of tabu list, (a) pair attribute- medium size (b) pair attribute- large size

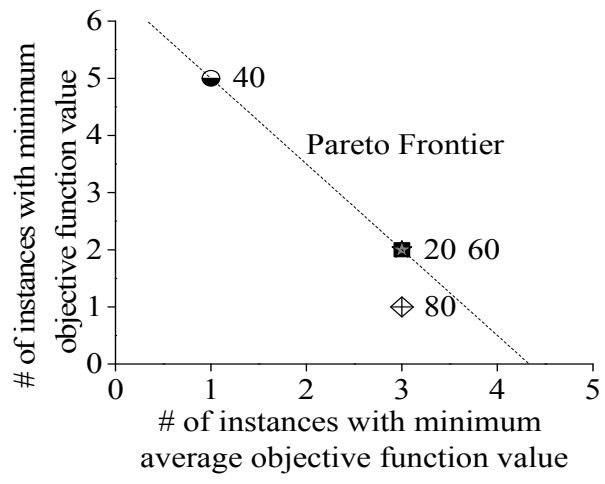

(a)



(b)

\subsection{Termination condition}

To determine the stopping rule of the CPTAP TS heuristic, we develop charts of the current and best-found solution values versus the iteration number for one medium size instance and one large size instance as shown in Figure 5. Interesting phenomena that are shown in both problem sizes are:

1 The current solution is generally worse (larger penalised cost function value) than the best solution found so far. It is likely that the current solution is frequently infeasible; therefore its objective function is penalised and larger than the best-found solution.

2 The best-found solution is improved dramatically in the first several hundreds of iterations. Then the improvement slows down with no practical improvement after 4,500 iterations. A number of local optimum can be identified through the 'big' steps of the best-found solutions. Based on the results shown in both charts of Figure 5, we set the final number of iterations to 5,000 to ensure a good-quality solution.

Figure 5 Heuristic termination analyses, (a) medium size (b) large size

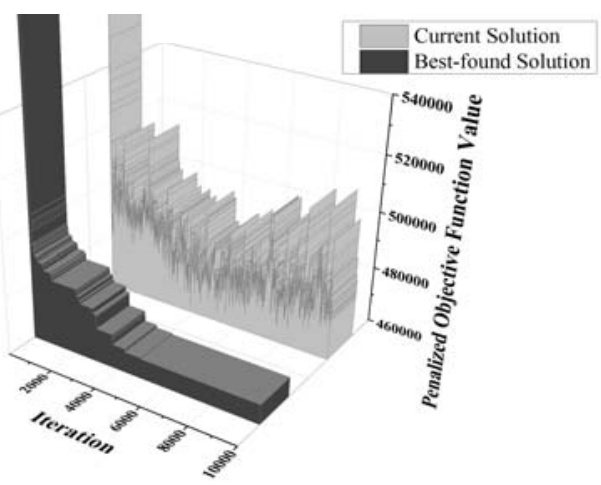

(a)

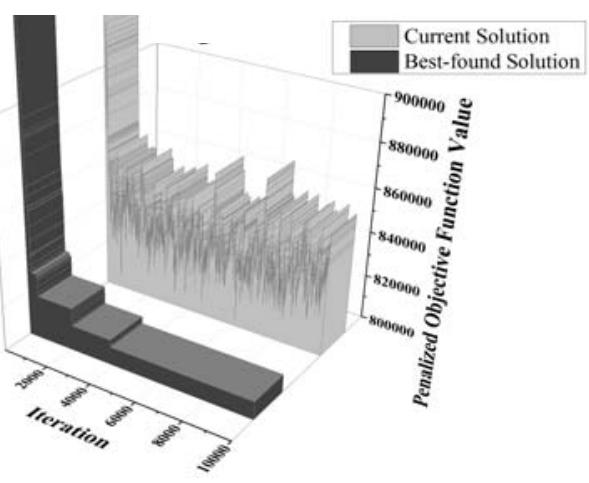

(b) 


\section{Computational results}

In this section, we determine the best TS heuristic variant and compare the resulting CPTAP TS heuristic with the benchmark results of the CPTAP GA method (Tong and Nachtmann, 2017). The CPTAP TS heuristic is coded in C++ language and run on a Dell Intel Core i7 CPU with 4.00GB of RAM.

In order to compare the two heuristics, we run the same instances that were generated previously for CPTAP GA-based heuristic (Tong and Nachtmann, 2017). The instances are systematically generated from a data set collected on the Upper Mississippi River. The study area is a 154-mile river section of the Upper Mississippi River, starting from Lock and Dam No.14 north of Davenport, Iowa (Moline, Illinois) to Lock and Dam No.19 at Keokuk, Iowa. Barge locations are generated uniformly over the study region according to the number of the terminals in the instance and the locations of the terminals. Water transport time of each barge from its current location to each terminal is calculated based on barge location, terminal location, and barge speed (assumed five miles per hour). With each pair of terminal and barge assignment, the offload time and the land transport time are estimated over uniform distributions of five to ten hours and 18 to 96 hours respectively. We assume barge volume to be 1000 per ton and the terminal capacity to be 5000 tons per commodity type. Two digit commodity type classification by USACE (2012b) is used as the cargo types and their 2012 tonnage data for the study region and the market price are displayed in Table 2 . We set the cargo type for each barge on the basis of the tonnage data and calculate the total cargo value on a barge by multiplying the barge volume and the market price. $100 \%$ of the petroleum and $50 \%$ of the chemicals are considered as the hazardous cargo. Barge draft (ranging from six to 14 feet) is determined according to its probability density function that is estimated from the draft data of vessel trips (USACE, 2012c). We assign one foot and $90 \%$ to safety level and sinking threshold respectively. Value decreasing rate for each commodity type is given as follows: Hazardous cargo receives the highest value decreasing rate $(\$ 600$ per 1,000 tons per hour) due to their high economic value and the dangerous feature to environment and people; nonhazardous chemicals and perishable cargo (food and form products) are given the second highest value decreasing rate ( $\$ 400$ per 1,000 tons per hour); the third highest value decreasing rate goes to crude materials and primary manufactured goods ( $\$ 300$ per 1,000 tons per hour); the lowest value decreasing rate is assigned to coal ( $\$ 100$ per 1,000 tons per hour) because of its stable value function. Problem size is defined as the sum of number of terminals and number of barges. Instances that fall into three problem size categories are investigated because problem size may be an influencing factor in the TS performance, which are small-size instances (five terminals and five/seven/nine barges), medium-size instances (ten terminals and thirty barges), and large-size instances (15 terminals and fifty barges). The CPTAP TS heuristic is run ten times for all the instances, which is in accordance with the CPTAP GA-based heuristic.

A summary of our CPTAP GA approach is described here, and more detail can be found in (Tong and Nachtmann, 2017). We first use a numerical string to represent a CPTAP solution. Natural numbers indicate the numbered barges and zeroes distinguish the terminals with the dummy terminal located at the end of the string. We employ tournament selection to choose two parent chromosomes in the population as the foundation to generate child chromosomes. The major components of the CPTAP GA are 
a crossover operator that produces two child chromosomes, a mutation operator that enables the GA to explore new solution areas, and a repair operator that restores the structurally infeasible chromosome(s) caused by the first two operators.

Table 2 Commodity types, tonnage data and market price on the study region

\begin{tabular}{lccc}
\hline Two-digit code & Cargo commodity type & Tonnage data & Market price \\
\hline 10 & Coal, lignite and coal coke & $10,288.25$ & $\$ 36.29 /$ ton \\
20 & Petroleum and petroleum products & $1,238.20$ & $\$ 403.39 /$ ton \\
30 & Chemicals and related product & $18,331.33$ & $\$ 399.88 /$ ton \\
40 & Crude materials, inedible, except & $11,364.99$ & $\$ 134.61 /$ ton \\
& fuels & & \\
50 & Primary manufactured goods & $7,843.58$ & $\$ 396.45 /$ ton \\
60 & Food and farm products & $58,670.63$ & $\$ 164.52 /$ ton \\
\hline
\end{tabular}

Source: USACE (2012b)

Table 3 exhibits our experimental results of the CPTAP TS heuristic under three different neighbourhood structures - partial shift (PS), blind SWAP (BS) and switch SWAP (SS). As previously mentioned, random generation is used for initial solution generation, and pair attribute is the tabu management scheme employed. Problem of sizes ten, twelve, and 14 are included in the small size problem experiments with five instances for each problem size. Experiments on ten instances of medium and large size problem are conducted. The optimal solutions (Opt) are presented in Table 3, which are found through the total enumeration program run on the high performance computers of the Arkansas High Performance Computing Center at the University of Arkansas. We can only determine optimal solutions for small size problems of size less than 14 due to the memory limit. The total value loss (Min) and the CPU time (CPU) of the CPTAP GA approach are expressed in dollars and seconds respectively in Table 3. Let $c\left(s^{G A}\right)$ and $c\left(s^{T S}\right)$ denote the total value loss of the CPTAP GA solution $s^{G A}$ and CPTAP TS solution $s^{T S}$. The estimated gap between the two heuristic results is given by

$$
\text { MinGap }=\frac{c\left(s^{T S}\right)-c\left(s^{G A}\right)}{c\left(s^{G A}\right)} \times 100 \%
$$

Similarly, we also calculate the CPU Gap of the two heuristics. TS-BS finds the same solution as the CPTAP GA for all the small size instances (two thirds are optimal), and TS-PS obtains the same solution as the CPTAP GA for 13 out of 15 small size instances. Both TS heuristics require higher CPU time with average gap of $36.6 \%$ for TS-PS and $515.8 \%$ for TS-BS.

A dramatic deterioration of the solution quality can be observed from the TS-SS over small size instances. TS-SS does not perform as well as the CPTAP GA on any of the 15 small size instances with a considerable higher total value loss of $10.73 \%$ on average. The poor comparative performance is anticipated to continue as problem size increases, and therefore TS-SS is excluded as a competitive alternative for the comparisons on medium and large size instances. When comparing TS-PS and CPTAP GA results for medium and large size problems, neither approach produces consistently better results than the other. On average, TS-PS yields higher total value loss than CPTAP by $0.31 \%$ on medium size instances but lower than the CPTAP GA on large size instances by 
$1.43 \%$. The computation time of TS-PS is substantially slower on average than the CPTAP GA for medium (784.95\%) and large (1162.14\%) size instances. TS-BS improves the total value loss produced by the CPTAP GA on all twenty instances of the medium and large size problems with $2.15 \%$ and $3.14 \%$ decreases on average. The average CPU time of TS-BS is higher on average than the CPTAP GA for medium $(384.96 \%)$ and large (192.11\%) size problem instances.

To summarise Table 3, TS-BS is identified as the best CPTAP TS heuristic. TS-BS is the only CPTAP TS variant that produces either as good as or improved solutions in all thirty-five experimental instances over the CPTAP GA. In fact, it produces the best-found result for all the medium and large size instances where optimal solutions cannot be obtained. In terms of the computational time, although TS-BS consumes more time than TS-PS for small size instances, its actual CPU time is as small as approximately one minute which definitely falls within an acceptable range. In medium and large size instances that more closely resemble realistic decision scenarios, TS-BS requires quite a bit less time than the TS-PS approach.

If we consider solution quality and computation time to compare the heuristic results in Table 3, the TS-BS and CPTAP GA do not dominate each other although TS-BS produces solutions with lower objective function values. In Figure 6, we show summarised computational results when reduce the number of iterations to 1,000 for the three CPTAP TS heuristic variants (TS-PS, TS-BS, and TS-SS) in order to make them comparable to the CPTAP GA (GA). For each heuristic scheme, average total value loss and CPU time of five instances are presented for both medium and large problem sizes. The best average total value loss is obtained by TS-BS in both problem sizes, which is in accordance with the previous discussion. Moreover, this heuristic generates the solution very quickly. For medium size instances, TS-BS computation time is slightly slower than TS-SS $(<1 \mathrm{~s})$ and faster than the other two heuristic variants $(+10 \mathrm{~s})$. Since TS-SS produces much higher average total value loss (approximately $\$ 95,000$ more than TS-BS), TS-BS is considered an overall better choice than TS-SS. Moreover, TS-BS is the fastest approach for solving large size problem instances. The other three heuristic variants require more time to obtain worse solutions when compared to TS-BS.

Figure 6 Quality vs. time of GA and TS heuristics for CPTAP, (a) medium size (b) large size



(a)

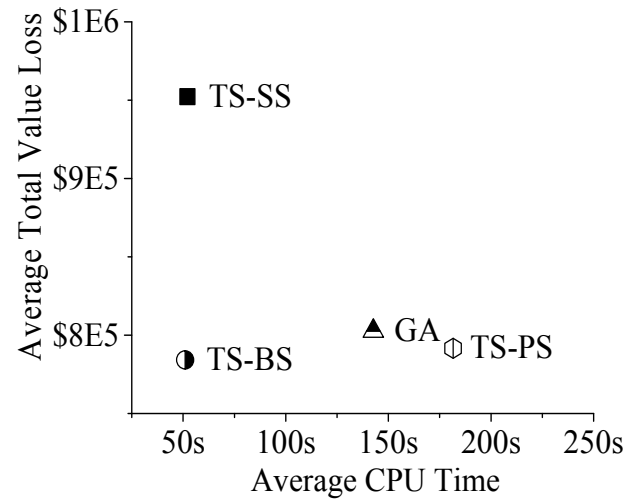

(b) 
Table 3 Experimental results of the three TS variants

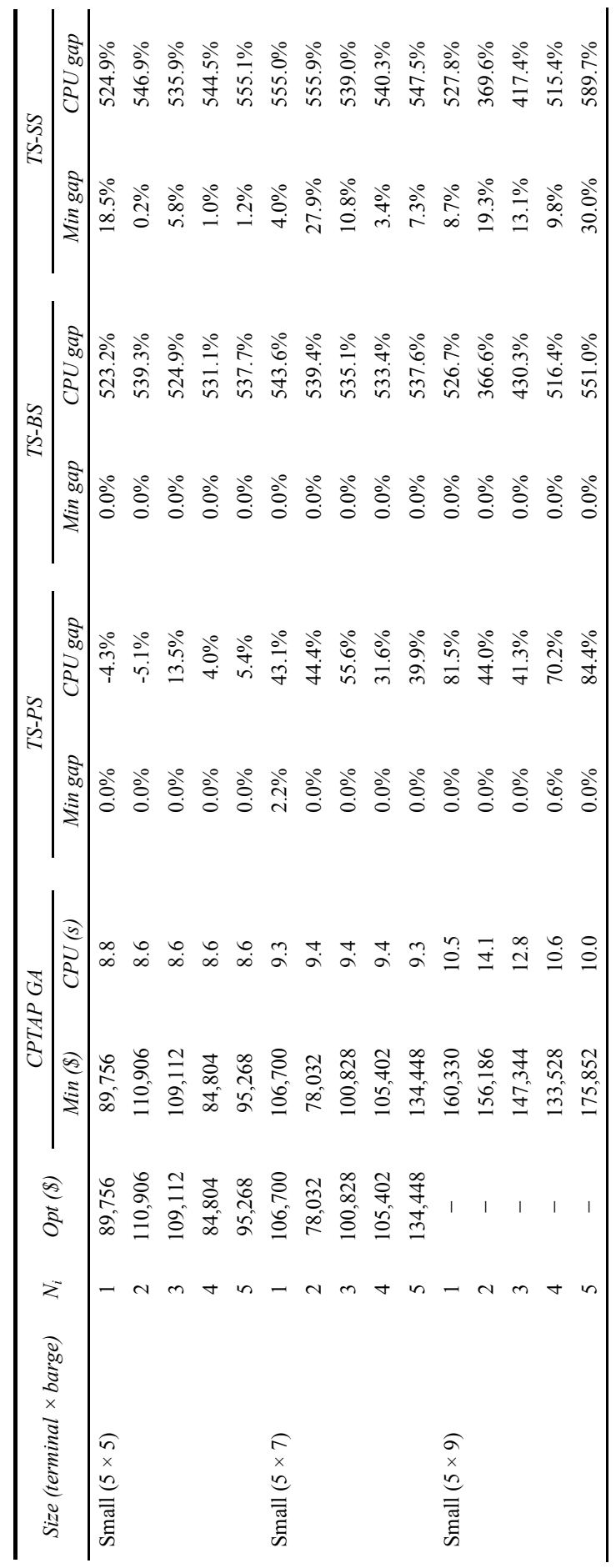


Table 3 Experimental results of the three TS variants (continued)

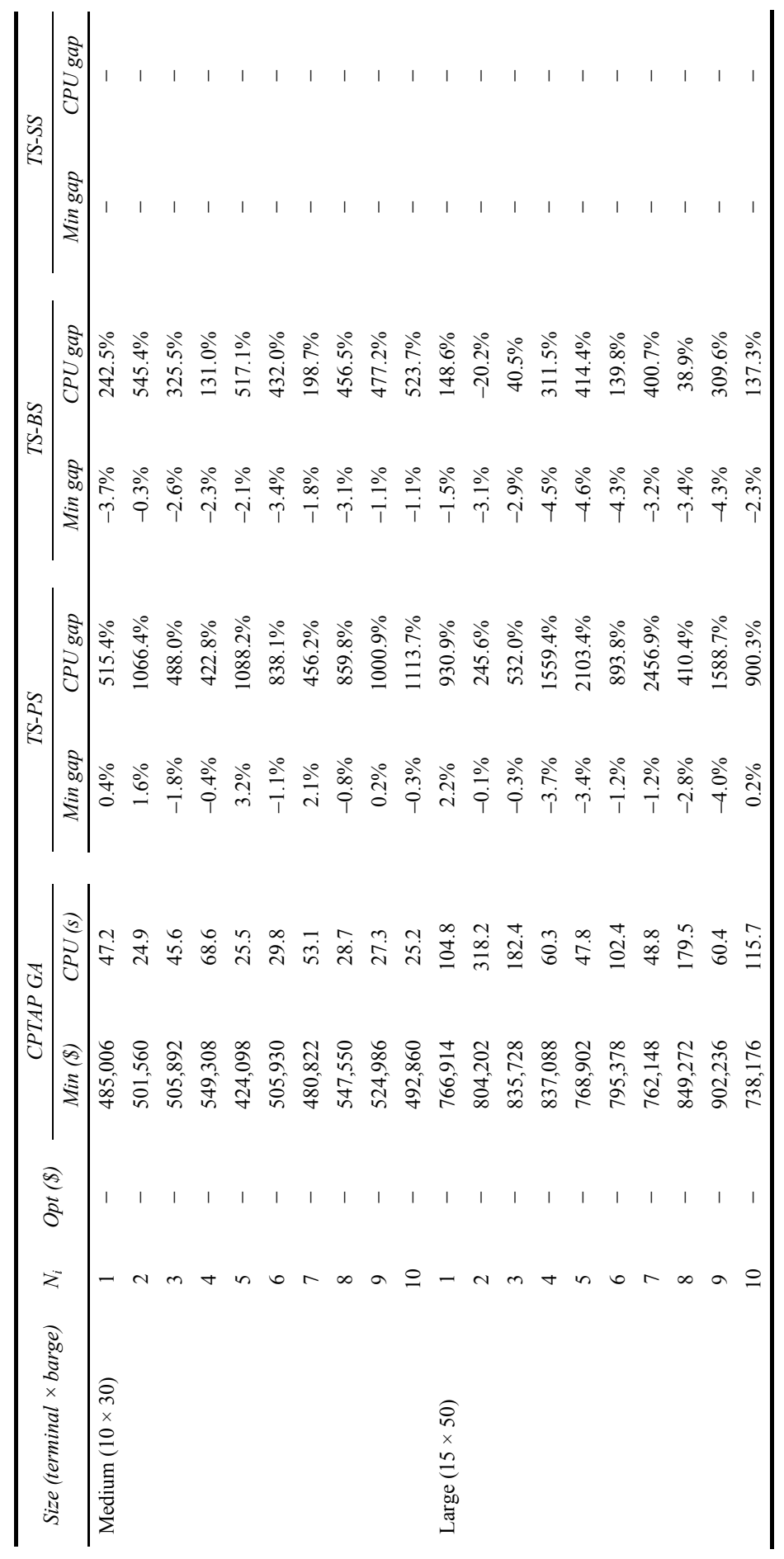




\section{CPTAP strategy comparison}

We further investigate the impact of applying CPTAP and its solution technique to realistic inland waterway disruption scenario. A naive approach to inland waterway disruption response is to assign the disrupted barge cargoes to their nearest feasible terminals. The objective function in this approach is to minimise the total distance of all disrupted barges transport to their assigned terminals. In this minimise distance (MD) approach, each terminal still serves one barge at a time, and every disrupted barge must be handled by a terminal. Capacity and draft constraints (constraint sets (6) and (7)) are again considered to ensure the barge can be accepted by the terminal. However, cargo type is not a critical factor in the MD strategy. Hazardous cargo is not being treated differently from nonhazardous cargo, and value loss does not influence the barge-terminal assignments. An integer programming model with linear objective function and constraints is developed to implement the MD approach and solved with AMPL-CPLEX. The formulation is provided as follows:

$u_{\text {in }}$ Offload capacity for cargo $n$ at terminal $i$ during the disruption response

$\underline{e}_{\text {jn }} 1$ if barge $j$ carries cargo $n ; 0$ otherwise

$c_{j} \quad$ cargo volume on barge $j$

$w_{i} \quad$ water depth at terminal $i$

$d_{j} \quad$ draft depth of barge $j$

$D_{i j}$ distance between terminal $i$ and barge $j$

$s$ safety level.

$$
\begin{aligned}
& \sum_{i \in I}^{\operatorname{Min}} \sum_{j \in J \cup H} \sum_{k \in K} D_{i j} x_{i j k} \\
& \sum_{i \in I} \sum_{k \in K} x_{i j k}=1 \quad \forall i \in J \cup H \\
& \sum_{j \in J \cup H} x_{i j k} \leq 1 \quad \forall i \in I, k \in K \\
& \sum_{j \in J \cup H} x_{i j k} \geq \sum_{j \in J \cup H} x_{i j(k+1)} \quad \forall i \in I, k \in K /|K| \\
& \sum_{j \in J \cup H} \sum_{k \in K} c_{j} e_{j n} x_{i j k} \leq u_{i n} \quad \forall i \in I, n \in N \\
& \sum_{i \in I} \sum_{k \in K}\left(w_{i}-d_{j}\right) x_{i j k} \geq s \quad \forall j \in J \cup H \\
& x_{i j k} \in\{0,1\} \quad \forall i \in I \cup D, k \in K, j \in J \cup H
\end{aligned}
$$

In Figure 7, we show the comparative results of three cargo prioritisation strategies (MD, CPTAP TS, and CPTAP GA) for two performance measures - total value loss and response time. Total value loss is the objective function value for the CPTAP TS and CPTAP GA approaches. For the MD approach, we calculate its total value loss after the 
optimal minimum distance barge-terminal assignments are determined. We compute the response time, the total time to complete water transport and cargo offloading of all disrupted barges, for all three approaches based on their optimal/best solutions. In disruption response, a rapidly-cleared river reduces the chance of secondary disaster and helps the maritime stakeholders resume transportation on the waterway as soon as possible. Therefore, a smaller response time is preferred when we select a cargo prioritisation strategy. According to Figure 7, the greater total value loss and response time are obtained when employing MD strategy as compared to CPTAP TS and GA strategies for all medium size instances. The CPTAP TS heuristic again produces a lower total value loss than the CPTAP GA-based heuristic in four out of the five medium instances and an equal value for the one instance. Prioritisation results from TS consumes less time than GA to transport all the disrupted barge cargoes off the waterway in three out of five instances and equal time to the GA in one instance. For large size instances, the TS results in the lowest total value loss and delivers the quickest response time for all the five large size instances. Overall, the results in Figure 7 indicate that involving cargo, barge and terminal features to intelligently prioritise the barge cargoes in CPTAP has a profound impact on the disruption response of inland waterway transportation, which mitigates the negative impacts of the disruption and provides an improved response towards waterway recovery, and the CPTAP TS method is again shown to perform better than the CPTAP GA-based heuristic according to the required response time.

Figure 7 Comparison of multiple strategies for cargo prioritization and terminal allocation,

(a) medium size (b) large size



(a)

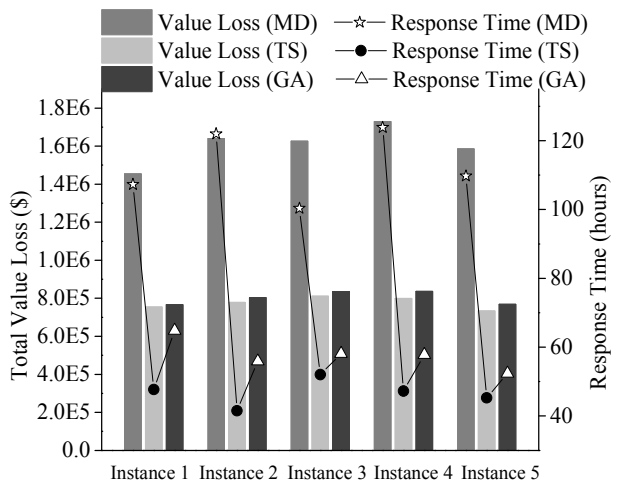

(b)

\section{Conclusions}

This paper developed a TS heuristic for a novel problem - cargo prioritisation and terminal allocation problem (CPTAP), which prioritises and reassigns cargo of a disrupted inland waterway transportation system (Tong and Nachtmann, 2017). We implemented and tested multiple TS variants on small, medium, and large size experimental instances and identified TS-BS as producing the best-quality results in a fastest manner. The TS-BS heuristic outperforms the previously recommended CPTAP GA approach, and our analysis indicates that CPTAP solved by either heuristic approach 
significantly decreases total value loss and response time compared to a naive prioritisation strategy based simply on assigning disrupted barges to the closest feasible terminal.

The contributions of this paper are twofold:

1 A robust TS heuristic that outperforms a previously developed GA approach to find improved solutions for CPTAP within a satisfactory amount of time.

2 The significance of applying CPTAP to disrupted inland waterway is systematically validated.

Both contributions are crucial steps that lead to our future research on improving CPTAP model and solution methods. Planned future work includes:

1 Application of the CPTAP TS heuristic during a real world CPTAP decision scenario to assess its implementation performance.

2 Development of a heuristic that incorporates merits from both population search (GA) and local search (TS).

3 Development of other potential solution approaches to CPTAP such as column generation.

\section{References}

American Society of Civil Engineers (ASCE) (2009) Infrastructure Fact Sheet [online] http://www.infrastructurereportcard.org/2009/sites/default/files/RC2009_inland_waterways.pd f (2009) (accessed 25 July 2014).

Bolduc, M.C., Laporte, G., Renaud, J. and Boctor, F.F. (2010) 'A tabu search heuristic for the split delivery vehicle routing problem with production and demand calendars', Eur. J. Oper. Res., Vol. 202, No. 1, pp.122-130.

Brandão, J. (2009) 'A deterministic tabu search algorithm for the fleet size and mix vehicle routing problem', Eur. J. Oper. Res., Vol. 195, No. 3, pp.716-728.

Bräysy, O. and Gendreau, M. (2005) 'Vehicle routing problem with time windows', Part II: Metaheuristics. Transport. Sci., Vol. 39, No. 1, pp.119-139.

Ceschia, S., Di Gaspero, L. and Schaerf, A. (2011) 'Tabu search techniques for the heterogeneous vehicle routing problem with time windows and carrier-dependent costs', J. Sched., Vol. 14, No. 6, pp.601-615.

Cordeau, J.F. and Laporte, G. (2004) 'Tabu search heuristics for the vehicle routing problem', in Rego, C. and Alidaee, B. (Eds.): Metaheuristic Optimization via Memory and Evolution: Tabu Search and Scatter Search, pp.145-163, Kluwer, Boston.

Cordeau, J.F., Gendreau, M. and Laporte, G. (1997) 'A tabu search heuristic for periodic and multi-depot vehicle routing problems', Networks, Vol. 30, No. 2, pp.105-119.

Cordeau, J.F., Gendreau, M., Laporte, G., Potvin, J.Y. and Semet, F. (2002) 'A guide to vehicle routing heuristics', J. Oper. Res. Soc., Vol. 53, No. 5, pp.512-522.

Cordeau, J.F., Laporte, G. and Mercier, A. (2001) 'A unified tabu search heuristic for vehicle routing problems with time windows', J. Oper. Res. Soc., Vol. 52, No. 8, pp.928-936.

Côté, J.F. and Potvin, J.Y. (2009) 'A tabu search heuristic for the vehicle routing problem with private fleet and common carrier', Eur. J. Oper. Res., Vol. 198, No. 2, pp.464-469.

Eksioglu, B., Vural, A.V. and Reisman, A. (2009) 'The vehicle routing problem: a taxonomic review', Comput. Ind. Eng., Vol. 57, No. 4, pp.1472-1483. 
Gendreau, M., Hertz, A. and Laporte, G. (1994) 'A tabu search heuristic for the vehicle routing problem', Manage. Sci., Vol. 40, No. 10, pp.1276-1290.

Gendreau, M., Iori, M., Laporte, G. and Martello, S. (2008) 'A tabu search heuristic for the vehicle routing problem with two-dimensional loading constraints', Networks, Vol. 51, No. 1, pp.4-18.

Giallombardo, G., Moccia, L., Salani, M. and Vacca, I. (2010) 'Modeling and solving the tactical berth allocation problem', Transport. Res. B-Meth., Vol. 44, No. 2, pp.232-245.

Imai, A., Nagaiwa, K.I. and Tat, C.W. (1997) 'Efficient planning of berth allocation for container terminals in Asia', J. Adv. Transport., Vol. 31, No. 1, pp.75-94.

Imai, A., Nishimura, E. and Papadimitriou, S. (2001) 'The dynamic berth allocation problem for a container port', Transport. Res. B-Meth., Vol. 35, No. 4, pp.401-417.

Imai, A., Nishimura, E. and Papadimitriou, S. (2003) 'Berth allocation with service priority', Transport. Res. B-Meth., Vol. 37, No. 5, pp.437-457.

Kim, K. and Moon, K. (2003) 'Berth scheduling by simulated annealing', Transport. Res. B-Meth., Vol. 37, No. 6, pp.541-560.

Laporte, G. (2009) 'Fifty years of vehicle routing', Transport. Sci., Vol. 43, No. 4, pp.408-416.

Magsam, J. and McGeeney, R. (2014) 'Haywire bridge stalls river, railroad traffic', Arkansas Democrat Gazette.

Meisel, F. and Bierwirth, C. (2009) 'Heuristics for the integration of crane productivity in the berth allocation problem', Transport. Res. E-Log., Vol. 45, No. 1, pp.196-209.

Nguyen, P.K., Crainic, T.G. and Toulouse, M. (2013) 'A tabu search for time-dependent multi-zone multi-trip vehicle routing problem with time windows', Eur. J. Oper. Res., Vol. 231, No. 1, pp.43-56.

Nishimura, E., Imai, A. and Papadimitriou, S. (2001) 'Berth allocation planning in the public berth system by genetic algorithms’, Eur. J. Oper. Res., Vol. 131, No. 2, pp.282-292.

Rochat, Y. and Taillard, É.D. (1995) 'Probabilistic diversification and intensification in local search for vehicle routing', J. Heuristics, Vol. 1, No. 1, pp.147-167.

Taillard, É.D., Gambardella, L.M., Gendreau, M. and Potvin, J.Y. (2001) 'Adaptive memory programming: a unified view of metaheuristics', Eur. J. Oper. Res., Vol. 135, No. 1, pp.1-16.

Tarantilis, C.D. (2005) 'Solving the vehicle routing problem with adaptive memory programming methodology', Comput. Oper. Res., Vol. 32, No. 9, pp.2309-2327.

Tong, J. and Nachtmann, H. (2017) 'Cargo prioritization and terminal allocation problem for inland waterway disruptions', Maritime Econ. and Log., Vol. 19, No. 3, pp.403-427.

US Army Corps of Engineers (USACE) (2012a) Inland Waterways and Export Opportunities [online] http://www.lrd.usace.army.mil/Portals/73/docs/Navigation/PCXIN/ Inland_Waterways_and_Export_Opportunities-FINAL_2013-01-03.pdf (accessed 15 April 2014).

US Army Corps of Engineers (USACE) (2012b) Commodity Classification List, Navigation Data Center [online] http://www.ndc.iwr.usace.army.mil/wcsc/webpub10/pdfs/commodity.pdf (accessed 18 April 2014).

US Army Corps of Engineers (USACE) (2012c) Vessel Trips by Waterways [online] http://www.navigationdatacenter.us/wcsc/webpub12/Part2_WWYs_Tripsbydraft_CY2012.HT M (accessed 18 April 2014).

US Army Corps of Engineers (USACE) (2013) The US Waterway System Transportation Facts and Information [online] http://www.navigationdatacenter.us/factcard/factcard13.pdf (accessed 20 March 2014).

US Army Corps of Engineers (USACE) (2014) Lock Performance Monitoring System [online] http://corpslocks.usace.army.mil/ (accessed 20 March 2014).

Wassan, N.A., Wassan, A.H. and Nagy, G. (2008) 'A reactive tabu search algorithm for the vehicle routing problem with simultaneous pickups and deliveries', J. Comb. Optim., Vol. 15, No. 4, pp.368-380. 


\section{Appendix}

Table A1 Sensitive analysis of adjustment factor $\delta$ - medium size

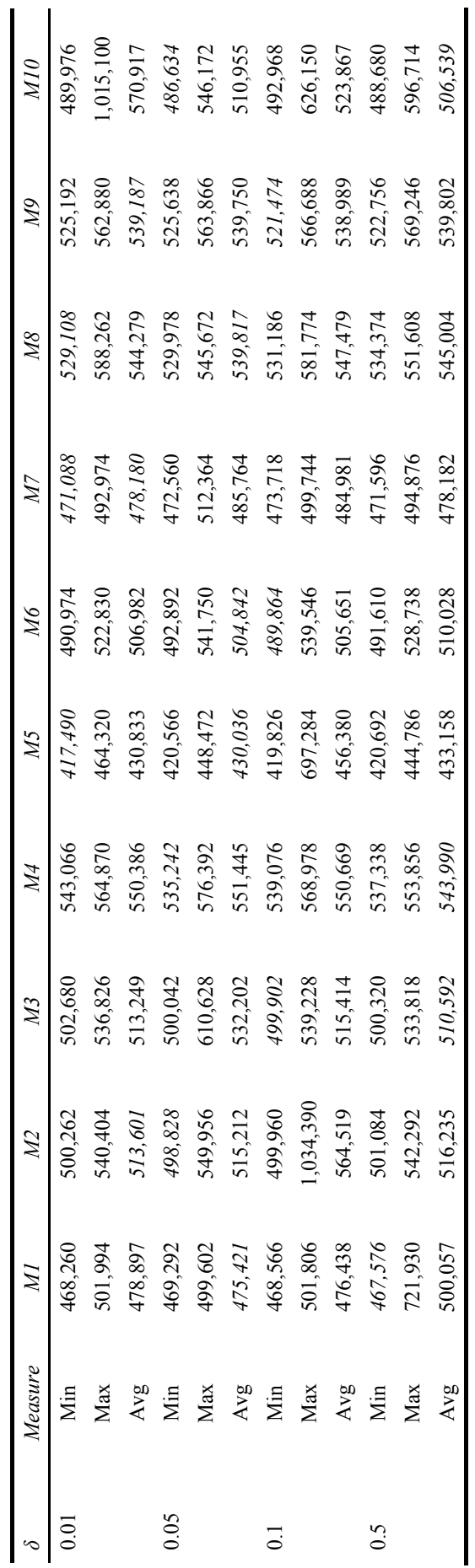


Table A2 Sensitive analysis of adjustment factor $\delta$ - large size

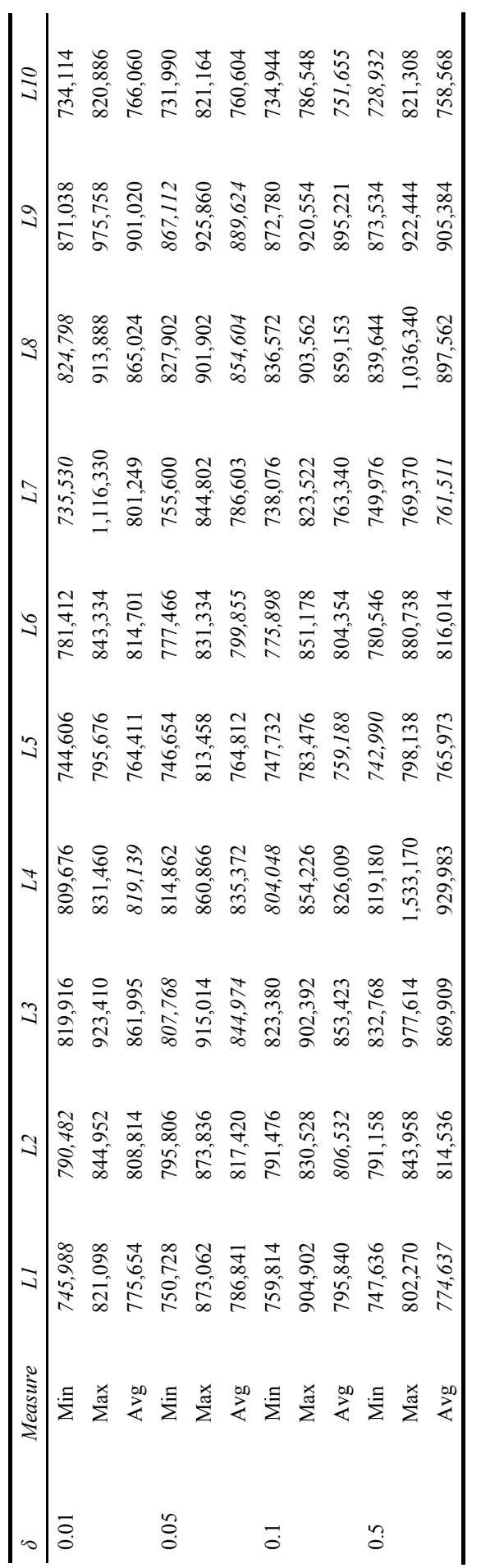

